\title{
Diagnostic, Predictive, Prognostic, and Therapeutic Molecular Biomarkers in Third Millennium: A Breakthrough in Gastric Cancer
}

\author{
Nicola Carlomagno, ${ }^{1}$ Paola Incollingo, ${ }^{1}$ Vincenzo Tammaro, ${ }^{1}$ Gaia Peluso, ${ }^{1}$ \\ Niccolò Rupealta, ${ }^{1}$ Gaetano Chiacchio, ${ }^{1}$ Maria Laura Sandoval Sotelo, ${ }^{1}$ Gianluca Minieri, ${ }^{1}$ \\ Antonio Pisani, ${ }^{2}$ Eleonora Riccio, ${ }^{2}$ Massimo Sabbatini, ${ }^{2}$ Umberto Marcello Bracale, \\ Armando Calogero, ${ }^{1}$ Concetta Anna Dodaro, ${ }^{1}$ and Michele Santangelo ${ }^{1}$
}

${ }^{1}$ Department of Advanced Biomedical Science, University of Naples Federico II, Via S. Pansini 5, 80131 Naples, Italy

${ }^{2}$ Department of Public Health, University of Naples Federico II, Via S. Pansini 5, 80131 Naples, Italy

Correspondence should be addressed to Paola Incollingo; paolaincollingo@hotmail.it

Received 7 April 2017; Accepted 12 July 2017; Published 28 September 2017

Academic Editor: Valeria Barresi

Copyright (C) 2017 Nicola Carlomagno et al. This is an open access article distributed under the Creative Commons Attribution License, which permits unrestricted use, distribution, and reproduction in any medium, provided the original work is properly cited.

\begin{abstract}
Introduction. Gastric cancer is the fifth most common cancer and the third cause of cancer death. The clinical outcomes of the patients are still not encouraging with a low rate of 5 years' survival. Often the disease is diagnosed at advanced stages and this obviously negatively affects patients outcomes. A deep understanding of molecular basis of gastric cancer can lead to the identification of diagnostic, predictive, prognostic, and therapeutic biomarkers. Main Body. This paper aims to give a global view on the molecular classification and mechanisms involved in the development of the tumour and on the biomarkers for gastric cancer. We discuss the role of E-cadherin, HER2, fibroblast growth factor receptor (FGFR), MET, human epidermal growth factor receptor (EGFR), hepatocyte growth factor receptor (HGFR), mammalian target of rapamycin (mTOR), microsatellite instability (MSI), PD-L1, and TP53. We have also considered in this manuscript new emerging biomarkers as matrix metalloproteases (MMPs), microRNAs, and long noncoding RNAs (lncRNAs). Conclusions. Identifying and validating diagnostic, prognostic, predictive, and therapeutic biomarkers will have a huge impact on patients outcomes as they will allow early detection of tumours and also guide the choice of a targeted therapy based on specific molecular features of the cancer.
\end{abstract}

\section{Introduction}

Gastric cancer is the fifth most common cancer after cancers of the lung, breast, colorectum, and prostate and it is the third cause of cancer death worldwide [1,2]. The clinical outcomes of patient affected by gastric cancer are still not encouraging; indeed the 5 years' survival is less than $30 \%$ [35]. The incidence of gastric cancer is wildly different among the countries. Even though Japan has a higher incidence it also has a higher survival rate (52\%) compared to other countries $[4,6,7]$.

In 1965, Laurén classification of gastric cancer was introduced; it divides cancer into two types: intestinal and diffuse types which seem to have a different pathogenesis. The intestinal type is characterized by a cohesive and expansive growth pattern, it consists of neoplastic intestinal glands similar to the intestinal adenocarcinoma. The age of incidence of the intestinal type is higher than the diffuse type; it occurs more often in males and is more often located in the antrum; it predominates in high risk areas and is preceded by precancerous lesions. It is associated with $H$. pylori infection that leads to atrophic gastritis and intestinal metaplasia (precursor of intestinal type gastric cancer) [811]. The diffuse type is characterized by an infiltrative and noncohesive growth pattern with single neoplastic cell or small group of cells widely infiltrating the gastric wall. It occurs in younger patients, with no significant difference 


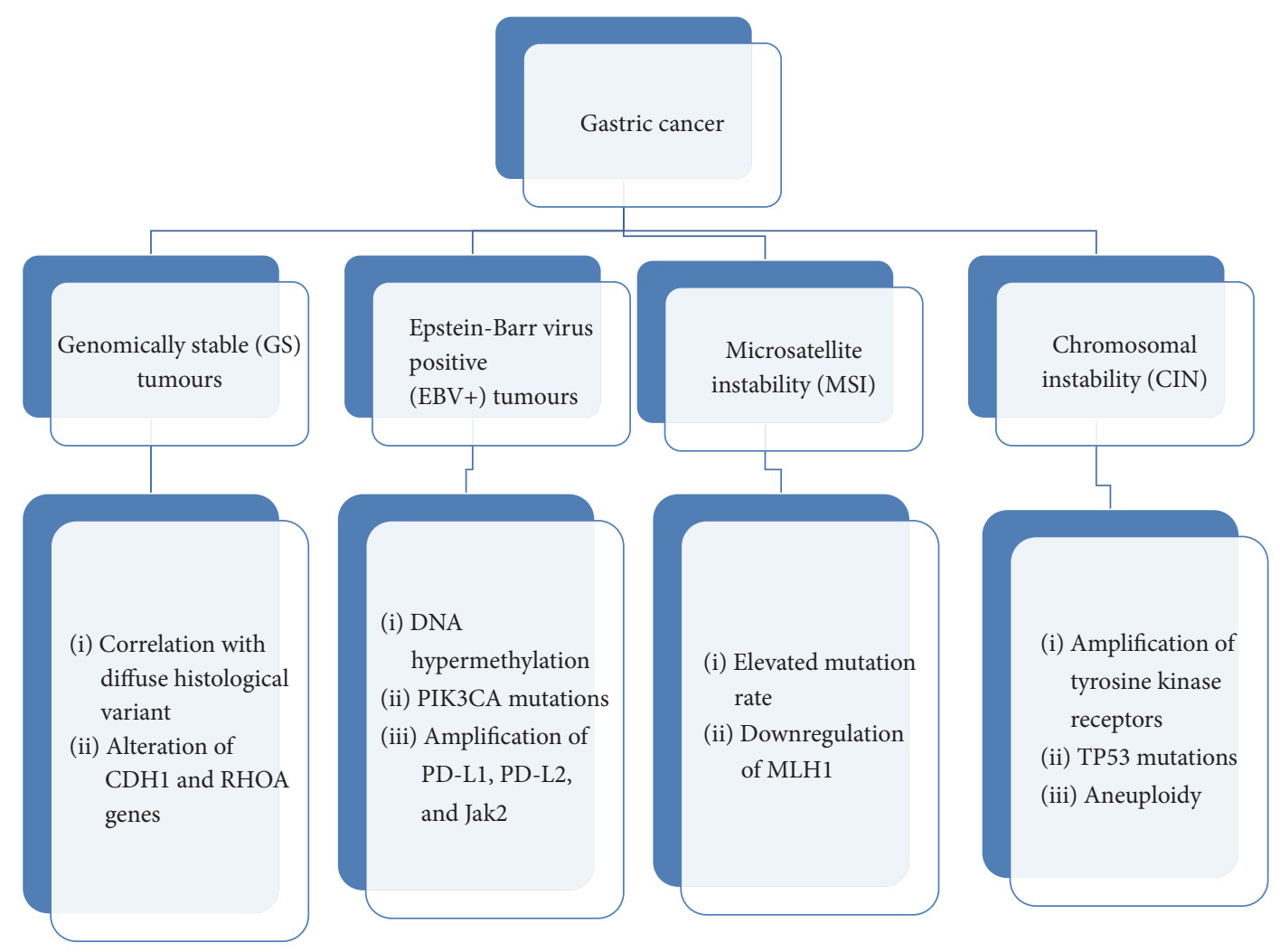

FIGURE 1: Classification of gastric cancers on the base of their molecular features (2014).

between men and women, and it is more often located in the gastric body $[11,12]$.

The TNM system is used for the staging of gastric cancer; however patients that belong to the same TNM stage often show very different clinical outcomes; this clearly manifests that there must be molecular factors responsible for those clinical differences.

A deep understanding of the molecular factors involved in the development of gastric cancer is needed in order to identify new biomarkers to diagnose GC in early stages and develop more effective therapeutic strategies.

In 2014 gastric cancer has been classified into four subtypes on the base of their molecular features: genomically stable (GS) tumours; tumours characterized by chromosomal instability (CIN); tumours positive for Epstein-Barr virus (EBV-positive); tumours characterized by microsatellite instability (MSI-positive). The GS tumours, nearly diploid tumours, are correlated to diffuse histological variant and alterations of genes CDH1 and RHOA. The CIN tumours are characterized by focal amplification of tyrosine kinase receptors, TP53 mutations, and aneuploidy. The Epstein-Barr positive tumours are associated with high levels of DNA hypermethylation, PIK3CA mutations, and amplification of CD274 (also known as $P D-L 1$ ) and PDCD1LG2 (also known as $P D-L 2)$ and JAK2. The MSI tumours display elevated mutation rate and downregulation of the MLH1 gene that codifies MLH1 protein involved in the mismatch repair (MMR) [13] (Figure 1).
It is essential to discover new biomarkers of gastric cancer that could lead to an early detection of the tumour or give predictive information about the response to a therapy and finally improve the therapeutic outcomes [14].

A valid biomarker for malignant tumour needs to have specific characteristics: it has to be detectable in high level in patient affected by cancer and undetectable or present in low level in people not affected; it has to be easily quantifiable in clinical sample; it has to show functions related to the progression of the disease and it has to provide prognostic or diagnostic information about the cancer $[15,16]$.

Biomarkers can be classified into four types: diagnostic, prognostic, predictive, and therapeutic. A diagnostic biomarker allows the early detection of the cancer in a noninvasive way and thus the secondary prevention of the cancer. A predictive biomarker allows predicting the response of the patient to a targeted therapy and so defining subpopulations of patients that are likely going to benefit from a specific therapy. A prognostic biomarker is a clinical or biological characteristic that provides information on the likely course of the disease; it gives information about the outcome of the patient $[14,17,18]$. A therapeutic biomarker is generally a protein that could be used as target for a therapy [18].

This paper aims to give a global view on the biomarkers for gastric cancer; we discuss the role of E-cadherin, HER2, fibroblast growth factor receptor (FGFR), MET, human epidermal growth factor receptor (EGFR), hepatocyte growth factor receptor (HGFR), mammalian target of rapamycin (mTOR), microsatellite instability (MSI), PD-L1, and TP53. 
We have also considered in this manuscript new emerging biomarkers as matrix metalloproteases (MMPs), microRNAs, and long noncoding RNAs (lncRNAs).

\section{E-Cadherin}

E-Cadherin is a transmembrane glycoprotein that is involved in the cellular calcium-mediated adhesion. It is codified by $\mathrm{CDH} 1$ located on the chromosome 16 (q22.1) [19, 20]. E-Cadherin plays a very important role in the adhesion and differentiation of the epithelial gastric cells and in the prevention of cancer onset [21]. CDH1 is one of the most important suppressor genes in gastric cancer; its inactivation increases tumour cells proliferation, invasion, and metastasis $[18,19,22-25]$. Several mechanisms can lead to loss of function of E-cadherin: mutations of the gene $\mathrm{CDH1}$, loss of heterozygosis (LOH), silencing through suppressors that bind the promoter of $\mathrm{CDH} 1$ or through hypermethylation, and microRNAs that control the E-cadherin expression [20].

Analysing family affected, many germline mutations have been identified in hereditary diffuse gastric cancer [26]. Those mutations are spread in the 16 exons of the $\mathrm{CDHl}$ gene; approximately $25 \%$ of them are missense mutations and $75 \%$ are truncating mutations $[27,28]$. Only in a little percentage of family affected by gastric cancer (4\%) have large deletions of $\mathrm{CDH} 1$ gene been identified $[29,30]$. In the $70 \%$ of HDGC families germline there is a monoallelic mutation of the gene $\mathrm{CDH} 1$ that leads to a Loss of Heterozygosity $(\mathrm{LOH})$ of the normal gene [31]. The cancer develops only when the second hit occurs, according to Knudson's model of the inactivation of tumour suppressor gene [31-34]. The second hit it is more often a hypermethylation of the gene's promoter and less often a second mutation or deletion occurring on the normal allele [21,35-37]. The gastric cancer manifests when the complete inactivation of the $\mathrm{CDH} 1$ gene occurs, leading to a lack of the E-cadherin expression $[27,38]$. Nowadays E-cadherin mutation cannot be considered a therapeutic biomarker as it would imply repairing E-cadherin expression through gene therapy [19]. Different E-cadherin alterations lead to various clinical manifestations and histotypes of gastric cancer so the presence of E-cadherin alteration is a weak prognostic biomarker [24]. A study showed a strong association between abnormal E-cadherin expression and tumour grade and metastases to regional lymph nodes [19]. Also another study showed the association between methylation of E-cadherin and dimension, stage of the cancer, and lymph nodes involvement [39]. Contrariwise another study did not find any association between E-cadherin mutation and gastric cancer stage and grade [40]. In most of the studies there is a correlation between E-cadherin abnormalities and worse clinical course, worse prognosis of the patient, and lower survival rate than patients negative for $\mathrm{CDH} 1$ mutations $[25,41]$.

E-Cadherin can also be considered as a predictive biomarker of the sensitivity to a specific therapy as its disablement reduces the response to both conventional and targeted therapy [24, 42]. Identifying $\mathrm{CDH} 1$ mutations at the moment of the diagnosis can predict if that cancer is going to be responsive to a therapy and so it could help in choosing the more suitable therapy for a specific patient [25] (Table 1).

It is important to highlight that a high percentage of families with HDGC have not got a mutation of E-cadherin gene; this obviously implies that there must be other molecular alterations that lead to the predisposition to gastric cancer and that still have not been identified [29, 43].

\section{Microsatellite Instability}

Microsatellites are short DNA repetitive sequences, in a nonrandom distribution along the human genome, that during DNA replication can lose out base-pairing mistakes [44, 45]. Those mistakes are normally repaired by the mismatch repair (MMR) proteins MLH1, MSH2, PMS2, and MSH6. Defects in the mismatch repair lead to a gathering of mutations that reflects the MSI and favour the onset of different types of cancer including the gastric one [46]. Several studies have reported an association between defects of mismatch repair and gastric cancer [47, 48]. MSI is observed in a percentage between 15 and 30 of all the gastric cancers and is more often due to hypermethylation of MLH1 promoter and the consequent lack of MLH1 expression [49-52]. MSI-positive gastric cancers show specific features: they usually have a later onset in life and are often located in distal part of the stomach and they usually have an intestinal histotype [45, 5356]. The MSI in patients affected by gastric cancer seems to be a positive prognostic factor [57]. MSI-positive tumours show a better prognosis compared to MSI-negative as they have a lower local invasion capacity and have a lower prevalence of lymph nodes involvement; they also have a higher survival rate compared to MSI-negative gastric cancer at the same stage $[50,55-60]$ (Table 1$)$.

\section{PD-L1}

PD-L1 and PD-L2 are ligands of Programmed Death-1 (PD1) that is an important checkpoint receptor involved in the regulation of immunity and tolerance mechanism of Tcell. PD-L1 binding PD-1 is responsible for inducing and keeping the tolerance of peripheral T-cells [57]. PD-L1 is overexpressed in about the $40 \%$ of gastric cancer belonging to the EBV-positive type $[13,61]$. Neoplastic cells use the PD1/PD-L1 pathway to escape the immune surveillance of T-cells and the immune system reply to the cancer $[62,63]$.

A monoclonal antibody anti-PD-1, Pembrolizumab, has manifested efficacy in patients affected by advanced gastric cancer, showing a six-month OS of 69\% [64]. The overexpression of $\mathrm{PD}-\mathrm{L} 1$ can then be considered as a predictive biomarker of the response to a targeted therapy (Table 1). Targeting the PD1/PD-L1 pathway represents a promising strategy for the treatment of GC $[57,65]$.

\section{TP53}

p53 is a nuclear protein that works as a transcriptional factor whose duty is to keep the genomic stability. When a damage of the DNA occurs, p53 binds the DNA and activates the transcription of genes responsible for stopping the cellular 
TABLE 1

\begin{tabular}{|c|c|c|}
\hline Biomarkers characteristics & Prognostic value & Predictive value \\
\hline $\begin{array}{l}\text { E-Cadherin } \\
\text { Transmembrane glycoprotein involved in calcium } \\
\text { mediated adhesion, codified by gene CDH1 } \\
\text { (chromosome } 16 \mathrm{q} 22.1 \text { ). } \\
\text { Its alterations lead to increased cell proliferation, } \\
\text { invasion, and metastasis. }\end{array}$ & $\begin{array}{l}\text { It is associated with worse prognosis and } \\
\text { lower survival rate }\end{array}$ & $\begin{array}{l}\text { It is associated with reduced } \\
\text { response to conventional and } \\
\text { targeted therapy }\end{array}$ \\
\hline
\end{tabular}

\section{Microsatellite instability}

Microsatellites are short repetitive sequences that can lose out base paring during replication. Defects in mismatch repair lead to MSI. Tumours with MSI usually have

(i) later onset

(ii) distal location

(iii) intestinal histotype.
MSI-positive cancers are associated with

(i) a better prognosis than MSI-negative

(ii) higher survival rate

(iii) lower local invasion capacity

(iv) lower prevalence of lymph nodes

involvement

\section{$P D-L 1$}

It is the ligand of Programmed Death-1; it is responsible for inducing and keeping tolerance of peripheral

T-cells. PD-1/PD-L1 pathway is used by neoplastic cell to escape immune surveillance.

TP53 There is a correlation between p53

P53 is a nuclear protein that works as a transcriptional

factor that activates apoptosis in case of DNA damage.

It is codified by TP53 (chromosome 17p13).

P53 alterations are often associated with CIN subtype.

\section{HER2}

It is a tyrosine receptor kinase (RTK) belonging to EGFR family, codified by ERBB2 (chromosome 17q21). It is involved in cell survival and proliferation.

HER2+ tumours are often located at the gastroesophageal junction and often associated with intestinal histotype.

\section{EGFR}

It belongs to the family of tyrosine kinase receptors. overexpression and tumour size.

The association with lymph nodes metastasis and shorter survival is still controversial
$\mathrm{PD}-\mathrm{L} 1$ overexpression is a predictive biomarker of response to Pembrolizumab
Still controversial: some studies report a more aggressive disease with worse prognosis but other studies do not confirm it
It is a predictive biomarker of the response to trastuzumab and lapatinib
It is associated with slightly differentiated, to high stage tumours and to a low survival
The use of anti-EGFR (cetuximab and panitumumab) associated with chemotherapy did not show any improvement in the clinical outcomes

Under evaluation

FGFR1-4

The fibroblast growth factor receptors belong to RTK

Under evaluation

family.

mTOR

The activation of many RTK induces the activation of

PIK3/mTOR pathway.

PIK3CA mutations frequently occur in EBV positive

PIK3CA mutation has been associated

with

Constitutive activation of

(i) worse prognosis

(ii) reduced survival rate

(iii) increased lymph nodes metastasis

cancers.

MET

It is a RKT belonging to the family of Hepatocyte Growth Factor Receptors (HGFR); it binds HGF/SF. Autophosphorylation of MET leads to the activation of downstream pathways responsible for cancer cells survival, proliferation, invasion, and metastasization.

It is associated with

(i) more aggressive disease

(ii) shorter survival
$\mathrm{PIK} 3 / \mathrm{mTOR}$ pathway is predictive of the response to Everolimus cycle and causing apoptosis of the cell. p53 is encoded by the gene TP53 located on the chromosome 17p13.1 [11, 66, 67]. The mechanisms leading to the damage of TP53 function are usually $\mathrm{LOH}$ and mutations and less often methylation [68]. TP53 mutation is frequently mutated in gastric cancer and it is reported in association with CIN subtype $[13,68]$. Heterogeneity of TP53 mutations in the same tumour is also reported as a result of multiple mutations of the gene [68]. Studies reported a higher prevalence of TP53 mutations in the intestinal type than in the diffuse type; another study instead reported a similar prevalence of TP53 mutations in the two types. Early and advanced intestinal type as well as advanced diffuse type show a high similar prevalence of TP53 mutations that are instead infrequent in early diffuse type of gastric
It is an important predictive biomarker of the response to rilotumumab 
cancer [68-72]. A correlation between p53 overexpression and size of the gastric cancer has been reported [73]. The association between p53 overexpression with lymph nodes metastasis and shorter survival is still controversial because it has been reported in some studies but not in others; therefore, at this moment in time, p53 cannot be considered a trustworthy prognostic biomarker [57, 68] (Table 1).

\section{HER2}

HER2 is one of the four tyrosine receptor kinases (RTKs) belonging to the family of EGFR (EGFR or HER1, HER2, HER3, and HER4); it is codified by the protooncogene ERBB2 located on chromosome 17q21 and plays an important role in cell survival and proliferation [14, 74].

For signal transmission HER2 needs to heterodimerize with another member of the HER family, mainly with EGFR [75]. The amplification of ERBB2 gene produces an overexpression of HER2 protein that leads to cancer cells survival, growth, and proliferation through the PI3K-AKT and the MAPK pathways [76, 77]. Overexpression of HER2 receptor as a prognostic and predictive biomarker, identified before in breast cancer, is becoming noticeable even in gastric cancer [57]. HER 2 overexpression has a variable incidence ranging from $9 \%$ to $38 \%$ in most of the studies, depending on the location of the cancer and on its histology [76, 7882]. The HER2 overexpression is more frequently observed in gastroesophageal junction tumours than in those with distal gastric location and it is more often associated with the intestinal type adenocarcinomas [83-90]. Cancers positive for HER2 overexpression are usually differentiated tumours [43, 80, 91]. ERBB2 gene mutation that leads to HER2 overexpression occurs in the early stage of carcinogenesis [92].

The role as a prognostic biomarker of HER2 is still doubtful; indeed some studies show an association of HER2 with a worse prognosis and a more aggressive disease; others contrariwise do not find a significant difference in prognosis between HER2 positive and HER2 negative cancers $[76,80-$ 82, 91, 93-103].

Still controversial is also the correlation between HER2 overexpression and clinical features of the tumour. Some studies indeed suggest an association of ERBB2 amplification with tumour size, lymph node metastasis, local invasion, and cancer stage; other studies instead do not find any link between them [85, 87-90, 95, 100, 102].

HER2 overexpression has become a very important predictive biomarker that allows clinicians to identify patients that are going to have a survival benefit from a biological therapy with the monoclonal antibody (trastuzumab) [104106].

The ToGa clinical phase 3 randomized controlled trial, conducted on patient affected by advanced gastric or gastroesophageal junction cancer, HER2-positive with an immunohistochemical 3+ score, compared the effectiveness of the association of trastuzumab and chemotherapy (cisplatin and a fluoropyrimidine) with the chemotherapy alone. The results of this study pointed out that patients treated with the association of trastuzumab and chemotherapy had a longer
OS (13.8 months versus 11.1) and even their progression free survival (PFS) was heightened compared to that of the patient treated only with chemotherapy [104].

At the moment, trastuzumab is the only targeted therapy permitted for advanced gastric cancer [107]. Other ways of blocking the HER2 receptor are now being researched.

Lapatinib is a tyrosine kinase inhibitor that blocks both HER2 and EGFR. A randomized phase III TyTAN trial compared the efficacy of the association lapatinib and paclitaxel with paclitaxel alone, in patients affected by HER2-positive advanced gastric cancer. The OS was of 11.0 months in patient treated with the association of lapatinib and paclitaxel and 8.9 months in the ones treated with paclitaxel alone and also the response rate was increased with the associated therapy, yet there was no significant difference in PFS [108].

Other HER2 targeted drugs such as neratinib and pertuzumab, whose efficacy on HER2-positive breast cancer has already been proved, have not been assessed yet on advanced gastric cancer in randomized clinical trials [57].

Ado-trastuzumab emtansine (T-DM1) is a drug composed of the monoclonal antibody trastuzumab linked to a cytotoxic drug on microtubules DM1. This conjugate efficacy has been evaluated in the phase II/III Gatsby, whose results have not been released yet, but ImmunoGen has revealed that they are not encouraging [109].

HER2 can then be considered as an important predictive biomarker that can guide the choice of the best therapy for the single patient (Table 1).

\section{EGFR}

Even EGFR belongs to the family of tyrosine kinase receptors. It was found to be overexpressed in about the $27 \%$ of gastric cancer and the incidence of the amplification of the gene was from $3 \%$ to $8 \%$ depending on the detection method used [110$112]$.

EGFR overexpression has been related to cancer histology slightly differentiated, low survival, and high stage [111].

Unfortunately, the use of targeted therapy anti-EGFR (cetuximab or panitumumab) together with chemotherapy did not show any improvement in the outcomes of the patients affected by advanced gastric cancer $[113,114]$ (Table 1).

Even inhibitors of tyrosine kinase (TKIs) have been considered as therapy in patients affected by advanced gastric cancer resistant to chemotherapy [78].

Unluckily, none of the studies has demonstrated a significant improvement of results compared to conventional therapy. Considering premises already made, further investigations are needed to identify subgroups of patients that might benefit from anti- EGFR therapies.

\section{FGFR}

FGFR1, FGFR2, FGFR3, and FGFR4 are fibroblast growth factor receptors belonging to the RTK family [14]. In 2012, Deng et al. reported that FGFR2 copy number gain was detected in $9 \%$ of cancers [110]. Considering the high expression of this receptor in some tumours, phase II studies are evaluating 
the efficacy of dovitinib (TKI258), a small FGFR2 inhibitor, on patients with FGFR2 amplification positive gastric cancer [110].

\section{9. mTOR}

The activation of many RTKs induces the activation of phosphatidylinositol-3-kinase (PIK3)/mTOR pathway. Mutations of the gene PIK3CA that codifies the alpha p110 catalytic subunit of PIK3 lead to constitutive activation of the PIK3/mTOR pathway $[14,115]$. PIK3CA mutation has been associated with a worse prognosis with reduced survival and increased lymph node metastasis $[14,116]$ (Table 1). The frequency of mutations varies from 5 to $67 \%$ in different studies [117-120]. PIK3CA mutations frequently occur in EBV-positive gastric cancer [119].

A mTOR inhibitor, Everolimus, has displayed potential benefit in advanced gastric cancer in phase II trials; however in phase III trials it did not lead to any significant rising of OS [121-123].

\section{MET}

MET is a RKT belonging to the family of hepatocyte growth factor receptor (HGFR), it binds HGF/SF (hepatocyte growth factor/scatter factor). Autophosphorylation of MET leads to the activation of a number of downstream pathways (PIK3, Akt, and RAS-MAPK) responsible for cancer cell survival, proliferation, invasion, and metastasization [124].

It is overexpressed in about $50 \%$ of advanced gastric cancer $[65,125]$.

MET gene overexpression is related to a bad prognosis; it is associated with a more aggressive disease, a shorter OS, and disease free survival compared to MET-negative gastric cancers [126-129].

It is also an important predictive biomarker. Rilotumumab is a monoclonal antibody able to prevent the binding of MET receptor and its ligand HGF; this targeted therapy in association with the chemotherapy improves the OS to 11.1 months in patient affected by a high level MET amplification cancer compared with 5.7 months of the patient that received the chemotherapy alone [130] (Table 1).

MET importance on carcinogenesis is becoming so evident that, nowadays, multiple studies are evaluating the efficacy of TKIs (like crizotinib and foretinib) on cancers with MET overexpression [125, 131].

\section{Promising Future Markers}

11.1. Matrix Metalloproteinase. The matrix metalloproteinases (MMPs) are a family of zinc-dependent endoproteinase whose function is to degrade the elements of the extracellular matrix [132]. Their work is regulated by the inhibitors of metalloproteinase (TIMPs) [133]. MMPs are involved in many physiological and also pathological processes [134]. MMPs have been found upregulated in gastric cancer and they have also been associated with specific pathological features of the cancer. Studies conducted on this subject prompt that MMPs and TIMP could be used as markers of peritoneal dissemination, depth of invasion, and metastasis [132].

Unfortunately, MMPs inhibitors have not demonstrated significant clinical benefit as therapy. In a clinical trial, conducted on patients affected by chemotherapy refractory advanced gastric cancer and gastroesophageal cancer, the MMP marimastat only determined a little difference in survival. The treatment was burdened by low tolerability and musculoskeletal pain [135]. Further studies about this subject are needed in order to identify the possible application of MMP in therapy of the advanced gastric cancer.

11.2. MicroRNA. MicroRNAs are 18 to 24 nucleotides noncoding RNA fragments whose function is to bind the $3^{\prime}$ UTR region of their target gene and regulate its expression by impairing the translation [136-138]. MicroRNAs are involved in the regulation of several process of the cell: proliferation, differentiation, migration, and invasion [136]. Many genes can be regulated just by a microRNA [139]. MicroRNAs seem to play a very important role in the carcinogenesis of gastric cancer; they can increase the expression of oncogenes or reduce the expression of tumour suppressor genes $[139,140]$.

Several microRNAs have been identified and recognized to be implicated in gastric cancer $[141,142]$. It is difficult to pick a miRNA as a cancer biomarker. Currently, there are no studies proving the effectiveness of miRNAs as predictive, prognostic or therapeutic biomarkers [57].

11.3. Long Noncoding RNAs. Long noncoding RNAs (lncRNAs) are sequences of nucleotides longer than 200 [143, 144]. Currently lncRNAs are catching researchers' attention because of an increasing amount of evidence suggesting that they play an important role in carcinogenesis and metastasis [139]. Nowadays about 135 lncRNAs have been recognized as altered in gastric cancer, so their potential role as diagnostic and prognostic markers has been speculated [143-145]. However, further studies about lncRNAs are needed in order to identify their possible clinical utilization.

\section{Conclusions}

Even if the incidence of gastric cancer reduced, it still remains the fifth most common cancer and it is characterized by negative prognosis and bad outcomes in response to chemotherapy. A deep understanding of molecular mechanisms of gastric carcinogenesis is essential to develop new therapeutic strategies and diagnostic, prognostic, and predictive biomarkers. The partition of gastric cancer into four molecular types (EBV-positive, MSI-positive, genomically stable, and chromosomal instability) allows dividing the patients on the basis of the molecular features of their cancer and identifying the best therapeutic approach [13]. A huge amount of studies has been conducted on molecular biomarkers; however the only predictive biomarker currently used is HER2 that allows identifying the patient that will benefit from a targeted therapy with trastuzumab. The majority of the patients still cannot be treated with a targeted therapy and nowadays still there are no diagnostic markers that can be used for secondary prevention. Most of 
the biomarkers till now identified still need to be validated before they can actually be employed in clinical practice [14]. Further studies that will identify and validate diagnostic, prognostic, predictive, and therapeutic biomarkers will have a huge impact on the outcomes of the patients, as they will allow the early detection of the tumour and also guide the choice of a targeted therapy based on the specific molecular features of the cancer [146-149].

\section{Conflicts of Interest}

The authors declare that there are no conflicts of interest regarding the publication of this article.

\section{References}

[1] Iarc 2016. GLOBOSCAN 2012: Stomach CancerEstimated Incidence, Mortality and Prevalence Worldwide in 2012.

[2] N. Carlomagno, M. L. Santangelo, B. Amato et al., "Total colectomy for cancer: Analysis of factors linked to patients' age," International Journal of Surgery, vol. 12, no. 2, pp. S135-S139, 2014.

[3] R. L. Siegel, K. D. Miller, and A. Jemal, "Cancer statistics, 2015," CA: Cancer Journal for Clinicians, vol. 65, no. 1, pp. 5-29, 2015.

[4] H. H. Hartgrink, E. P. Jansen, N. C. van Grieken, and C. J. van de Velde, "Gastric cancer," The Lancet, vol. 374, no. 9688, pp. 477-490, 2009.

[5] M. Santangelo, A. Esposito, V. Tammaro et al., "What indication, morbidity and mortality for central pancreatectomy in oncological surgery? A systematic review," International Journal of Surgery, vol. 28, pp. S172-S176, 2016.

[6] A. Ohtsu, "Chemotherapy for metastatic gastric cancer: past, present, and future," Journal of Gastroenterology, vol. 43, no. 4, pp. 256-264, 2008.

[7] M. L. Santangelo, C. Criscitiello, A. Renda et al., "Immunosuppression and Multiple Primary Malignancies in Kidney-Transplanted Patients: A Single-Institute Study," BioMed Research International, vol. 2015, Article ID 183523, 2015.

[8] S. S. Kim, V. E. Ruiz, J. D. Carroll, and S. F. Moss, "Helicobacter pylori in the pathogenesis of gastric cancer and gastric lymphoma," Cancer Letters, vol. 305, no. 2, pp. 228-238, 2011.

[9] S. F. Moss and P. Malfertheiner, "Helicobacter and gastric malignancies," Helicobacter, vol. 12, supplement 1, pp. 23-30, 2007.

[10] S. Ming, "Cellular and molecular pathology of gastric carcinoma and precursor lesions: a critical review," Gastric Cancer, vol. 1, no. 1, pp. 31-50, 1998.

[11] V. Kumar, A. Abbas, and N. Fausto, Robins and Cotran: Pathologic Basis of Disease, Elsevier Masson, Issy-les-Moulineaux, France, 7th edition, 2006.

[12] G. Y. Lauwers, Odze and Goldblum Surgical Pathology of the GI Tract, Liver, Biliary Tract and Pancreas, Elsevier, Saunders, Philadelphia, Pennsylvania, 3rd edition, 2015.

[13] Cancer Genome Atlas Research Network, "Comprehensive molecular characterization of gastric adenocarcinoma," Nature, vol. 513, no. 7517, pp. 202-209, 2014.

[14] C. Durães, G. M. Almeida, R. Seruca, C. Oliveira, and F. Carneiro, "Biomarkers for gastric cancer: prognostic, predictive or targets of therapy?" Virchows Archiv, vol. 464, no. 3, pp. 367$378,2014$.
[15] S. Gilad, E. Meiri, Y. Yogev et al., "Serum microRNAs are promising novel biomarkers," PLoS ONE, vol. 3, no. 9, Article ID e3148, 2008.

[16] A. Etheridge, I. Lee, L. Hood, D. Galas, and K. Wang, "Extracellular microRNA: a new source of biomarkers," Mutation Research, vol. 717, no. 1-2, pp. 85-90, 2011.

[17] A. Italiano, "Prognostic or predictive? It's time to get back to definitions!," Journal of Clinical Oncology, vol. 29, no. 35, article 4718, 2011.

[18] L.-L. Lin, H.-C. Huang, and H.-F. Juan, "Discovery of biomarkers for gastric cancer: a proteomics approach," Journal of Proteomics, vol. 75, no. 11, pp. 3081-3097, 2012.

[19] R. Anbiaee, K. M. Sheibani, P. Torbati et al., "Abnormal expression of E-cadherin in gastric adenocarcinoma and its correlation with tumor histopathology and helicobacter pylori infection," Iranian Red Crescent Medical Journal, vol. 15, no. 3, pp. 218-222, 2013.

[20] P. Carneiro, M. S. Fernandes, J. Figueiredo et al., "E-cadherin dysfunction in gastric cancer-cellular consequences, clinical applications and open questions," FEBS Letters, vol. 586, no. 18, pp. 2981-2989, 2012.

[21] G. Tamura, "Alterations of tumor suppressor and tumor-related genes in the development and progression of gastric cancer," World Journal of Gastroenterology, vol. 12, no. 2, pp. 192-198, 2006.

[22] A. O. O. Chan, "E-cadherin in gastric cancer," World Journal of Gastroenterology, vol. 12, no. 2, pp. 199-203, 2006.

[23] G. Christofori and H. Semb, "The role of the cell-adhesion molecule E-cadherin as a tumour-suppressor gene," Trends in Biochemical Sciences, vol. 24, no. 2, pp. 73-76, 1999.

[24] P. Ferreira, M. J. Oliveira, E. Beraldi et al., "Loss of functional E-cadherin renders cells more resistant to the apoptotic agent taxol in vitro," Experimental Cell Research, vol. 310, no. 1, pp. 99104, 2005.

[25] G. Corso, J. Carvalho, D. Marrelli et al., "Somatic mutations and deletions of the e-cadherin gene predict poor survival of patients with gastric cancer," Journal of Clinical Oncology, vol. 31, no. 7, pp. 868-875, 2013.

[26] V. R. Blair, "Familial gastric cancer: genetics, diagnosis, and management," Surgical Oncology Clinics of North America, vol. 21, no. 1, pp. 35-56, 2012.

[27] M. Barber, A. Murrell, Y. Ito et al., "Mechanisms and sequelae of E-cadherin silencing in hereditary diffuse gastric cancer," Journal of Pathology, vol. 216, no. 3, pp. 295-306, 2008.

[28] K. Schrader and D. Huntsman, "Hereditary diffuse gastric cancer," Cancer Treatment and Research, vol. 155, pp. 33-63, 2010.

[29] H. Pinheiro, R. Bordeira-Carriço, S. Seixas et al., "Allele-specific CDH1 downregulation and hereditary diffuse gastric cancer," Human Molecular Genetics, vol. 19, no. 5, pp. 943-952, 2010.

[30] C. Oliveira, J. Senz, P. Kaurah et al., "Germline CDH1 deletions in hereditary diffuse gastric cancer families," Human Molecular Genetics, vol. 18, no. 9, pp. 1545-1555, 2009.

[31] A. G. Knudson, "Two genetic hits (more or less) to cancer," Nature Reviews Cancer, vol. 1, no. 2, pp. 157-162, 2001.

[32] A. G. Knudson Jr., "Mutation and cancer: statistical study of retinoblastoma," Proceedings of the National Academy of Sciences of the United States of America, vol. 68, no. 4, pp. 820-823, 1971.

[33] A. G. Knudson, "Cancer genetics," American Journal of Medical Genetics, vol. 111, no. 1, pp. 96-102, 2002. 
[34] A. G. Knudson Jr., "Retinoblastoma: a prototypic Hereditary neoplasm," Seminars in Oncology, vol. 5, no. 1, pp. 57-60, 1978.

[35] C. Oliveira, S. Sousa, H. Pinheiro et al., "Quantification of epigenetic and genetic 2nd hits in $\mathrm{CDH} 1$ during hereditary diffuse gastric cancer syndrome progression," Gastroenterology, vol. 136, no. 7, pp. 2137-2148, 2009.

[36] C. Oliveira, J. de Bruin, S. Nabais et al., "Intragenic deletion of $\mathrm{CDH} 1$ as the inactivating mechanism of the wild-type allele in an HDGC tumour," Oncogene, vol. 23, no. 12, pp. 2236-2240, 2004.

[37] W. M. Grady, J. Willis, P. J. Guilford et al., "Methylation of the $\mathrm{CDH} 1$ promoter as the second genetic hit in hereditary diffuse gastric cancer," Nature Genetics, vol. 26, no. 1, pp. 16-17, 2000.

[38] F. Carneiro, D. G. Huntsman, T. C. Smyrk et al., "Model of the early development of diffuse gastric cancer in E-cadherin mutation carriers and its implications for patient screening," Journal of Pathology, vol. 203, no. 2, pp. 681-687, 2004.

[39] F. Graziano, F. Arduini, A. Ruzzo et al., "Prognostic analysis of E-cadherin gene promoter hypermethylation in patients with surgically resected, node-positive, diffuse gastric cancer," Clinical Cancer Research, vol. 10, no. 8, pp. 2784-2789, 2004.

[40] A. Jawhari, S. Jordan, S. Poole, P. Browne, M. Pignatelli, and M. J. G. Farthing, "Abnormal immunoreactivity of the E-cadherincatenin complex in gastric carcinoma: relationship with patient survival," Gastroenterology, vol. 112, no. 1, pp. 46-54, 1997.

[41] A. Gamboa-Dominguez, C. Dominguez-Fonseca, Y. ChavarriGuerra et al., "E-cadherin expression in sporadic gastric cancer from Mexico: exon 8 and 9 deletions are infrequent events associated with poor survival," Human Pathology, vol. 36, no. 1, pp. 29-35, 2005.

[42] H. W. Xin, J. H. Yang, and D. M. Nguyen, "Sensitivity to epidermal growth factor receptor tyrosine kinase inhibitor requires E-cadherin in esophageal cancer and malignant pleural mesothelioma," Anticancer Research, vol. 33, no. 6, pp. 24012408, 2013.

[43] H. T. Lynch, W. Grady, G. Suriano, and D. Huntsman, "Gastric cancer: new genetic developments," Journal of Surgical Oncology, vol. 90, no. 3, pp. 114-133, 2005.

[44] T. Jascur and C. R. Boland, "Structure and function of the components of the human DNA mismatch repair system," International Journal of Cancer, vol. 119, no. 9, pp. 2030-2035, 2006.

[45] U. Shokal and P. C. Sharma, "Implication of microsatellite instability in human gastric cancers," Indian Journal of Medical Research, vol. 135, no. 5, pp. 599-613, 2012.

[46] Y. Maehara, A. Egashira, E. Oki, Y. Kakeji, and T. Tsuzuki, "DNA repair dysfunction in gastrointestinal tract cancers," Cancer Science, vol. 29, no. 3, pp. 451-458, 2008.

[47] G. Corso, C. Pedrazzani, D. Marrelli, V. Pascale, E. Pinto, and F. Roviello, "Correlation of microsatellite instability at multiple loci with long-term survival in advanced gastric carcinoma," Archives of Surgery, vol. 144, no. 8, pp. 722-727, 2009.

[48] W. K. Leung, J. J. Kim, J. G. Kim, D. Y. Graham, and A. R. Sepulveda, "Microsatellite instability in gastric intestinal metaplasia in patients with and without gastric cancer," The American journal of pathology, vol. 156, no. 2, pp. 537-543, 2000.

[49] S. Oda, Y. Zhao, and Y. Maehara, "Microsatellite instability in gastrointestinal tract cancers: a brief update," Surgery Today, vol. 35, no. 12, pp. 1005-1015, 2005.

[50] S. Velho, M. S. Fernandes, M. Leite, C. Figueiredo, and R. Seruca, "Causes and consequences of microsatellite instability in gastric carcinogenesis," World Journal of Gastroenterology, vol. 20, no. 44, pp. 16433-16442, 2014.

[51] G. Corso, S. Velho, J. Paredes et al., "Oncogenic mutations in gastric cancer with microsatellite instability," European Journal of Cancer, vol. 47, no. 3, pp. 443-451, 2011.

[52] S. Y. Leung, S. T. Yuen, L. P. Chung, K. M. Chu, A. S. Y. Chan, and J. C. I. Ho, "hMLH1 promoter methylation and lack of hMLH1 expression in sporadic gastric carcinomas with high-frequency microsatellite instability," Cancer Research, vol. 59, no. 1, pp. 159$164,1999$.

[53] S. H. Kim, B. K. Ahn, Y. S. Nam, J. Y. Pyo, Y. H. Oh, and K. H. Lee, "Microsatellite instability is associated with the clinicopathologic features of gastric cancer in sporadic gastric cancer patients," Journal of Gastric Cancer, vol. 10, no. 4, pp. 149$154,2010$.

[54] T. Nakajima, Y. Akiyama, J. Shiraishi et al., "Age-related hypermethylation of the hMLH1 promoter in gastric cancers," International Journal of Cancer, vol. 94, no. 2, pp. 208-211, 2001.

[55] C. Oliveira, R. Seruca, M. Seixas, and M. Sobrinho-Simões, "The clinicopathological features of gastric carcinomas with microsatellite instability may be mediated by mutations of different 'target genes': a study of the TGF $\beta$ RII, IGFII R, and BAX genes," American Journal of Pathology, vol. 153, no. 4, pp. 1211-1219, 1998.

[56] C. Pedrazzani, G. Corso, S. Velho et al., "Evidence of tumor microsatellite instability in gastric cancer with familial aggregation," Familial Cancer, vol. 8, no. 3, pp. 215-220, 2009.

[57] N. Baniak, J.-L. Senger, S. Ahmed, S. C. Kanthan, and R. Kanthan, "Gastric biomarkers: a global review," World Journal of Surgical Oncology, vol. 14, no. 1, article 212, 2016.

[58] N. R. Dos Santos, R. Seruca, M. Constancia, M. Seixas, and M. Sobrinho-Simoes, "Microsatellite instability at multiple loci in gastric carcinoma: clinicopathologic implications and prognosis," Gastroenterology, vol. 110, no. 1, pp. 38-44, 1996.

[59] R. Seruca, N. R. Santos, L. David et al., "Sporadic gastric carcinomas with microsatellite instability display a particular clinicopathologic profile," International Journal of Cancer, vol. 64, no. 1, pp. 32-36, 1995.

[60] S. Beghelli, G. De Manzoni, S. Barbi et al., "Microsatellite instability in gastric cancer is associated with better prognosis in only stage II cancers," Surgery, vol. 139, no. 3, pp. 347-356, 2006.

[61] A. G. Raufi and S. J. Klempner, "Immunotherapy for advanced gastric and esophageal cancer: preclinical rationale and ongoing clinical investigations," Journal of Gastrointestinal Oncology, vol. 6, no. 5, pp. 561-569, 2015.

[62] A. H. Sharpe, E. J. Wherry, R. Ahmed, and G. J. Freeman, "The function of programmed cell death 1 and its ligands in regulating autoimmunity and infection," Nature Immunology, vol. 8, no. 3, pp. 239-245, 2007.

[63] B. T. Fife and K. E. Pauken, "The role of the PD-1 pathway in autoimmunity and peripheral tolerance," Annals of the New York Academy of Sciences, vol. 1217, no. 1, pp. 45-59, 2011.

[64] K. Muro, Y. Bang, V. Shankaran et al., "Relationship between PD-L1 expression and clinical outcomes in patients (Pts) with advanced gastric cancer treated with the anti-PD-1 monoclonal antibody pembrolizumab (Pembro; MK-3475) in KEYNOTE012," Journal of Clinical Oncology, vol. 33, supplement 3, 2015.

[65] I. Panarese, F. De Vita, A. Ronchi et al., "Predictive biomarkers along gastric cancer pathogenetic pathways," Expert Review of Anticancer Therapy, vol. 17, no. 5, pp. 417-425, 2017. 
[66] M. C. Liu and E. P. Gelmann, "P53 gene mutations: case study of a clinical marker for solid tumors," Seminars in Oncology, vol. 29, no. 3, pp. 246-257, 2002.

[67] V. A. Belyi, P. Ak, E. Markert et al., "The origins and evolution of the p53 family of genes," Cold Spring Harbor perspectives in biology, vol. 2, no. 6, Article ID a001198, 2010.

[68] M. F. Bellini, A. C. T. Cadamuro, M. Succi, M. A. Proença, and A. E. Silva, "Alterations of the TP53 gene in gastric and esophageal carcinogenesis," Journal of Biomedicine and Biotechnology, vol. 2012, Article ID 891961, 13 pages, 2012.

[69] E. Oki, Y. Zhao, R. Yoshida et al., "The difference in p53 mutations between cancers of the upper and lower gastrointestinal tract," Digestion, vol. 79, no. 1, pp. 33-39, 2009.

[70] H. Iwamatsu, K. Nishikura, H. Watanabe et al., "Heterogeneity of 533 mutational status in the superficial spreading type of early gastric carcinoma," Gastric Cancer, vol. 4, no. 1, pp. 20-26, 2001.

[71] C. M. Fenoglio-Preiser, J. Wang, G. N. Stemmermann, and A. Noffsinger, "TP53 and gastric carcinoma: a review," Human Mutation, vol. 21, no. 3, pp. 258-270, 2003.

[72] X. P. Liu, K. Tsushimi, M. Tsushimi et al., "Expression of p53 protein as a prognostic indicator of reduced survival time in diffuse-type gastric carcinoma," Pathology International, vol. 51, no. 6, pp. 440-444, 2001.

[73] H. Z. Lu, J. P. Wu, W. Luo et al., "Correlation between aneuploidy of chromosome 17, over- expression of TP53 and TOP-II alpha and the clinicophatological features and diagnosis of adenocarcinoma," ZhonghuaZhong Liu ZaZhi, vol. 31, no. 10, pp. 754-758, 2009.

[74] N. Normanno, C. Bianco, L. Strizzi et al., "The ErbB receptors and their ligands in cancer: an overview," Current Drug Targets, vol. 6, no. 3, pp. 243-257, 2005.

[75] S.-H. I. Ou, "Second-generation irreversible epidermal growth factor receptor (EGFR) tyrosine kinase inhibitors (TKIs): a better mousetrap? A review of the clinical evidence," Critical Reviews in Oncology/Hematology, vol. 83, no. 3, pp. 407-421, 2012.

[76] C. Gravalos and A. Jimeno, "HER2 in gastric cancer: a new prognostic factor and a novel therapeutic target," Annals of Oncology, vol. 19, no. 9, pp. 1523-1529, 2008.

[77] A. Gallardo, E. Lerma, D. Escuin et al., "Increased signalling of EGFR and IGF1R, and deregulation of PTEN/PI3K/Akt pathway are related with trastuzumab resistance in HER2 breast carcinomas," British Journal of Cancer, vol. 106, no. 8, pp. 13671373, 2012.

[78] T. Dragovich, S. McCoy, C. M. Fenoglio-Preiser et al., "Phase II trial of erlotinib in gastroesophageal junction and gastric adenocarcinomas: SWOG 0127," Journal of Clinical Oncology, vol. 24, no. 30, pp. 4922-4927, 2006.

[79] A. F. C. Okines and D. Cunningham, "Trastuzumab in gastric cancer," European Journal of Cancer, vol. 46, no. 11, pp. 19491959, 2010.

[80] B. E. Phillips, R. R. Tubbs, T. W. Rice et al., "Clinicopathologic features and treatment outcomes of patients with human epidermal growth factor receptor 2-positive adenocarcinoma of the esophagus and gastroesophageal junction," Diseases of the Esophagus, vol. 26, no. 3, pp. 299-304, 2013.

[81] D. S. Chan, F. Campbell, P. Edwards, B. Jasani, G. T. Williams, and W. G. Lewis, "Relative prognostic value of human epidermal growth factor receptor 2 (HER2) expression in operable oesophagogastric cancer," ISRN Surgery, vol. 2012, Article ID 804891, 6 pages, 2012.
[82] A. F. C. Okines, L. C. Thompson, D. Cunningham et al., "Effect of HER2 on prognosis and benefit from peri-operative chemotherapy in early oesophago-gastric adenocarcinoma in the MAGIC trial," Annals of Oncology, vol. 24, no. 5, pp. 12531261, 2013.

[83] NCNN guidelines gastric cancer, Principles of systemic therapy (GAST-E), 2012, http://www.ncnn.org/professionals/physician_gls/f_guidelines.asp\#site.

[84] J. E. Boers, H. Meeuwissen, and N. Methorst, "HER2 status in gastro-oesophageal adenocarcinomas assessed by two rabbit monoclonal antibodies (SP3 and 4B5) and two in situ hybridization methods (FISH and SISH)," Histopathology, vol. 58, no. 3, pp. 383-394, 2011.

[85] Y. Kimura, E. Oki, A. Yoshida et al., "Significance of accurate human epidermal growth factor receptor-2 (HER2) evaluation as a new biomarker in gastric cancer," Anticancer Research, vol. 34, no. 8, pp. 4207-4212, 2014.

[86] P. L. Kunz, A. Mojtahed, G. A. Fisher et al., "HER2 expression in gastric and gastroesophageal junction adenocarcinoma in a US population: clinicopathologic analysis with proposed approach to HER2 assessment," Applied Immunohistochemistry and Molecular Morphology, vol. 20, no. 1, pp. 13-24, 2012.

[87] M. A. Kim, E. J. Jung, H. S. Lee et al., "Evaluation of HER-2 gene status in gastric carcinoma using immunohistochemistry, fluorescence in situ hybridization, and real-time quantitative polymerase chain reaction," Human Pathology, vol. 38, no. 9, pp. 1386-1393, 2007.

[88] A. H. Marx, L. Tharun, J. Muth et al., "HER-2 amplification is highly homogenous in gastric cancer," Human Pathology, vol. 40, no. 6, pp. 769-777, 2009.

[89] S. Y. Yan, Y. Hu, J. G. Fan et al., "Clinicopathologic significance of HER-2/neu protein expression and gene amplification in gastric carcinoma," World Journal of Gastroenterology, vol. 17, no. 11, pp. 1501-1506, 2011.

[90] C. B. Moelans, A. N. Milne, F. H. Morsink, G. J. A. Offerhaus, and P. J. van Diest, "Low frequency of HER2 amplification and overexpression in early onset gastric cancer," Cellular Oncology, vol. 34, no. 2, pp. 89-95, 2011.

[91] S. B. Fisher, K. E. Fisher, M. H. Squires III et al., "HER2 in resected gastric cancer: is there prognostic value?" Journal of Surgical Oncology, vol. 109, no. 2, pp. 61-66, 2014.

[92] M. Fassan, L. Mastracci, F. Grillo et al., "Early HER2 dysregulation in gastric and oesophageal carcinogenesis," Histopathology, vol. 61, no. 5, pp. 769-776, 2012.

[93] M. D. Begnami, E. Fukuda, J. H. T. G. Fregnani et al., "Prognostic implications of altered human epidermal growth factor receptors (HERs) in gastric carcinomas: HER2 and HER3 are predictors of poor outcome," Journal of Clinical Oncology, vol. 29, no. 22, pp. 3030-3036, 2011.

[94] H. Grabsch, S. Sivakumar, S. Gray, H. E. Gabbert, and W. Müller, "HER2 expression in gastric cancer: rare, heterogeneous and of no prognostic value-conclusions from 924 cases of two independent series," Cellular Oncology, vol. 32, no. 1-2, pp. 5765, 2010.

[95] F. Zhou, N. Li, W. Jiang et al., "Prognosis significance of HER$2 /$ neu overexpression/amplification in Chinese patients with curatively resected gastric cancer after the ToGA clinical trial," World Journal of Surgical Oncology, vol. 10, article 274, 2012.

[96] M. Terashima, K. Kitada, A. Ochiai et al., "Impact of expression of human epidermal growth factor receptors EGFR and ERBB2 on survival in stage II/III gastric cancer," Clinical Cancer Research, vol. 18, no. 21, pp. 5992-6000, 2012. 
[97] M. Aizawa, A. K. Nagatsuma, K. Kitada et al., "Evaluation of HER2-based biology in 1,006 cases of gastric cancer in a Japanese population," Gastric Cancer, vol. 17, no. 1, pp. 34-42, 2014.

[98] Y. Y. Janjigian, D. Werner, C. Pauligk et al., "Prognosis of metastatic gastric and gastroesophageal junction cancer by HER2 status: a European and USA International collaborative analysis," Annals of Oncology, vol. 23, no. 10, pp. 2656-2662, 2012.

[99] B. Yan, E. X. Yau, S. S. B. Omar et al., "A study of HER2 gene amplification and protein expression in gastric cancer," Journal of Clinical Pathology, vol. 63, no. 9, pp. 839-842, 2010.

[100] M. Tanner, M. Hollmen, and T. T. Junttila, "Amplification of HER-2 in gastric carcinoma: association with topoisomerase II $\alpha$ gene amplification, intestinal type, poor prognosis and sensitivity to trastuzumab," Annals of Oncology, vol. 16, pp. 273278, 2005.

[101] G. Bar-Sela, D. Hershkovitz, N. Haim, O. Kaidar-Person, K. Shulman, and O. Ben-Izhak, "The incidence and prognostic value of HER2 overexpression and cyclin D1 expression in patients with gastric or gastroesophageal junction adenocarcinoma in Israel," Oncology Letters, vol. 5, no. 2, pp. 559-563, 2013.

[102] D. I. Park, J. W. Yun, J. H. Park et al., "HER-2/neu amplification is an independent prognostic factor in gastric cancer," Digestive Diseases and Sciences, vol. 51, no. 8, pp. 1371-1379, 2006.

[103] Y. Kataoka, H. Okabe, A. Yoshizawa et al., "HER2 expression and its clinicopathological features in resectable gastric cancer," Gastric Cancer, vol. 16, no. 1, pp. 84-93, 2013.

[104] Y.-J. Bang, E. Van Cutsem, A. Feyereislova et al., “Trastuzumab in combination with chemotherapy versus chemotherapy alone for treatment of HER2-positive advanced gastric or gastrooesophageal junction cancer (ToGA): a phase 3, open-label, randomised controlled trial," The Lancet, vol. 376, no. 9742, pp. 687-697, 2010.

[105] C. A. Hudis, "Trastuzumab-mechanism of action and use in clinical practice," The New England Journal of Medicine, vol. 357, no. 1, pp. 39-51, 2007.

[106] J. J. M. Boone, J. Bhosle, M. J. Tilby, J. A. Hartley, and D. Hochhauser, "Involvement of the HER2 pathway in repair of DNA damage produced by chemotherapeutic agents," Molecular Cancer Therapeutics, vol. 8, no. 11, pp. 3015-3023, 2009.

[107] C. Gomez-Martín, F. Lopez-Rios, J. Aparicio et al., "A critical review of HER2-positive gastric cancer evaluation and treatment: from trastuzumab, and beyond," Cancer Letters, vol. 351, no. 1, pp. 30-40, 2014.

[108] T. Satoh, R. H. Xu, H. C. Chung et al., "Lapatinib plus paclitaxel versus paclitaxel alone in the second-line treatment of HER2-amplified advanced gastric cancer in Asian populations: TyTAN_a randomized, phase III study," Journal of Clinical Oncology: Official Journal of the American Society of Clinical Oncology, vol. 32, no. 19, pp. 2039-2049, 2014.

[109] a study of trastuzumabemtansine versus taxane in patiens with advanced gastric cancer, http://clinicaltrials.gov/ct2/show/ NTC0164.

[110] N. Deng, L. K. Goh, H. Wang et al., "A comprehensive survey of genomic alterations in gastric cancer reveals systematic patterns of molecular exclusivity and co-occurrence among distinct therapeutic targets," Gut, vol. 61, no. 5, pp. 673-684, 2012.

[111] M. A. Kim, H. S. Lee, H. E. Lee, Y. K. Jeon, H. K. Yang, and W. H. Kim, "EGFR in gastric carcinomas: prognostic significance of protein overexpression and high gene copy number," Histopathology, vol. 52, no. 6, pp. 738-746, 2008.
[112] S.-I. Kiyose, K. Nagura, H. Tao et al., "Detection of kinase amplifications in gastric cancer archives using fluorescence in situ hybridization," Pathology International, vol. 62, no. 7, pp. 477-484, 2012.

[113] F. Lordick, Y. K. Kang, H. C. Chung et al., "Capecitabine and cisplatin with or without cetuximab for patients with previously untreated advanced gastric cancer (EXPAND): a randomized, open-label Phase 3 trial," The Lancet Oncology, vol. 13, article 490, 2013.

[114] T. Waddell, I. Chau, D. Cunningham et al., "Epirubicin, oxaliplatin, and capecitabine with or without panitumumab for patients with previously untreated advanced oesophagogastric cancer (REAL3): a randomised, open-label phase 3 trial," The Lancet Oncology, vol. 14, no. 6, pp. 481-489, 2013.

[115] S. S. Singh, W. N. Yap, F. Arfuso et al., "Targeting the PI3K/Akt signaling pathway in gastric carcinoma: a reality for personalized medicine?" World Journal of Gastroenterology, vol. 21, no. 43, pp. 12261-12273, 2015.

[116] G. Yu, J. Wang, Y. Chen et al., "Overexpression of phosphorylated mammalian target of rapamycin predicts lymph node metastasis and prognosis of chinese patients with gastric cancer," Clinical Cancer Research, vol. 15, no. 5, pp. 1821-1829, 2009.

[117] S. Velho, C. Oliveira, A. Ferreira et al., "The prevalence of PIK3CA mutations in gastric and colon cancer," European Journal of Cancer, vol. 41, no. 11, pp. 1649-1654, 2005.

[118] J. Shi, D. Yao, W. Liu et al., "Highly frequent PIK3CA amplification is associated with poor prognosis in gastric cancer," $B M C$ Cancer, vol. 12, article 50, 2012.

[119] J. Lee, P. van Hummelen, C. Go et al., "High-throughput mutation profiling identifies frequent somatic mutations in advanced gastric Adenocarcinoma," PLoS ONE, vol. 7, no. 6, Article ID e38892, 2012.

[120] S. Barbi, I. Cataldo, G. De Manzoni et al., "The analysis of PIK3CA mutations in gastric carcinoma and metanalysis of literature suggest that exon-selectivity is a signature of cancer type," Journal of Experimental and Clinical Cancer Research, vol. 29, no. 1, article 32, 2010.

[121] D. H. Yoon, M.-H. Ryu, Y. S. Park et al., "Phase II study of everolimus with biomarker exploration in patients with advanced gastric cancer refractory to chemotherapy including fluoropyrimidine and platinum," British Journal of Cancer, vol. 106, no. 6, pp. 1039-1044, 2012.

[122] T. Doi, K. Muro, N. Boku et al., "Multicenter phase II study of everolimus in patients with previously treated metastatic gastric cancer," Journal of Clinical Oncology, vol. 28, no. 11, pp. 19041910, 2010.

[123] A. Ohtsu, J. A. Ajani, Y.-X. Bai et al., "Everolimus for previously treated advanced gastric cancer: results of the randomized, double-blind, phase III GRANITE-1 study," Journal of Clinical Oncology, vol. 31, no. 31, pp. 3935-3943, 2013.

[124] A. Z. Lai, J. V. Abella, and M. Park, "Crosstalk in Met receptor oncogenesis," Trends in Cell Biology, vol. 19, no. 10, pp. 542-551, 2009.

[125] J. K. Lennerz, E. L. Kwak, A. Ackerman et al., "MET amplification identifies a small and aggressive subgroup of esophagogastric adenocarcinoma with evidence of responsiveness to crizotinib," Journal of Clinical Oncology, vol. 29, no. 36, pp. 4803-4810, 2011.

[126] R. Erichsen, K. S. Oliner, M. A. Kelsh et al., "Prognostic impact of tumor MET expression among patients with stage IV gastric 
cancer: a Danish cohort study," Journal of Clinical Oncology, vol. 32, supplement 3, pp. 43-43, 2014.

[127] A. Shedev and DV. Cartacci, "Gastroesophageal cancer: focus on epidemiology, classification, and staging," Discov Med, vol. 16, no. 87, pp. 103-11, 2013.

[128] Y. Y. Janjigian, L. H. Tang, D. G. Coit et al., "MET expression and amplification in patients with localized gastric cancer," Cancer Epidemiology Biomarkers and Prevention, vol. 20, no. 5, pp. 10211027, 2011

[129] J. Lee, J. W. Seo, H. J. Jun et al., "Impact of MET amplification on gastric cancer: possible roles as a novel prognostic marker and a potential therapeutic target," Oncology Reports, vol. 25, no. 6, pp. 1517-1524, 2011.

[130] M. Zhu, R. Tang, S. Doshi et al., "Exposure-response analysis of rilotumumab in gastric cancer: the role of tumour MET expression," British Journal of Cancer, vol. 112, no. 3, pp. 429437, 2015.

[131] M. A. Shah, Z. A. Wainberg, D. V. T. Catenacci et al., "Phase II study evaluating 2 dosing schedules of oral foretinib (GSK1363089), cMET/VEGFR2 inhibitor, in patients with metastatic gastric cancer," PLoS ONE, vol. 8, no. 3, Article ID e54014, 2013.

[132] C. L. Sampieri, K. León-Córdoba, and J. M. Remes-Troche, "Matrix metalloproteinases and their tissue inhibitors in gastric cancer as molecular markers," Journal of Cancer Research and Therapeutics, vol. 9, no. 3, pp. 356-363, 2013.

[133] A. H. Baker, D. R. Edwards, and G. Murphy, "Metalloproteinase inhibitors: biological actions and therapeutic opportunities," Journal of Cell Science, vol. 115, no. 19, pp. 3719-3727, 2002.

[134] S. Swarnakar, S. Paul, L. P. Singh, and R. J. Reiter, "Matrix metalloproteinases in health and disease: regulation by melatonin," Journal of Pineal Research, vol. 50, no. 1, pp. 8-20, 2011.

[135] S. R. Bramhall, M. T. Hallissey, J. Whiting et al., "Marimastat as maintenance therapy for patients with advanced gastric cancer: a randomised trial," British Journal of Cancer, vol. 86, no. 12, pp. 1864-1870, 2002.

[136] T.-S. Han, K. Hur, G. Xu et al., "MicroRNA-29c mediates initiation of gastric carcinogenesis by directly targeting ITGB1," Gut, vol. 64, no. 2, pp. 203-214, 2015.

[137] Z.-X. Su, J. Zhao, Z.-H. Rong, Y.-G. Wu, W.-M. Geng, and C.-K. Qin, "Diagnostic and prognostic value of circulating miR-18a in the plasma of patients with gastric cancer," Tumor Biology, vol. 35, no. 12, pp. 12119-12125, 2014.

[138] L. Xu, Y. Hou, G. Tu et al., "Nuclear Drosha enhances cell invasion via an EGFR-ERK1/2-MMP7 signaling pathway induced by dysregulated miRNA-622/197 and their targets LAMC2 and CD82 in gastric cancer," Cell Death and Disease, vol. 8, no. 3, Article ID e2642, 2017.

[139] X. Zhu, M. Lv, H. Wang, and W. Guan, "Identification of circulating microRNAs as novel potential biomarkers for gastric cancer detection: a systematic review and meta-analysis," Digestive Diseases and Sciences, vol. 59, no. 5, pp. 911-919, 2014.

[140] Z. Zhu, X. Zhang, G. Wang, and H. Zheng, "Role of MicroRNAs in hepatocellular carcinoma," Hepatitis Monthly, vol. 14, no. 8, Article ID e18672, 2014.

[141] H.-H. Wu, W.-C. Lin, and K.-W. Tsai, "Advances in molecular biomarkers for gastric cancer: miRNAs as emerging novel cancer markers," Expert Reviews in Molecular Medicine, vol. 16, article el, 2014.

[142] H.-S. Liu and H.-S. Xiao, "MicroRNAs as potential biomarkers for gastric cancer," World Journal of Gastroenterology, vol. 20, no. 34, pp. 12007-12017, 2014.
[143] X.-Y. Fang, H.-F. Pan, R.-X. Leng, and D.-Q. Ye, "Long noncoding RNAs: novel insights into gastric cancer," Cancer Letters, vol. 356, no. 2, pp. 357-366, 2015.

[144] M. U. Kaikkonen, M. T. Y. Lam, and C. K. Glass, "Noncoding RNAs as regulators of gene expression and epigenetics," Cardiovascular Research, vol. 90, no. 3, pp. 430-440, 2011.

[145] H. Song, W. Sun, G. Ye et al., "Long non-coding RNA expression profile in human gastric cancer and its clinical significances," Journal of Translational Medicine, vol. 11, no. 1, article 225, 2013.

[146] I. Riquelme, K. Saavedra, J. A. Espinoza et al., "Molecular classification of gastric cancer: towards a pathwaydriven targeted therapy," Oncotarget, vol. 6, no. 28, pp. 24750-24779, 2015.

[147] N. Carlomagno, F. Schonauer, V. Tammaro, A. Di Martino, C. Criscitiello, and M. L. Santangelo, "A multidisciplinary approach to an unusual medical case of locally advanced gastric cancer: a case report," Journal of medical case reports, vol. 9, p. 13, 2015.

[148] M. L. Santangelo, C. Grifasi, C. Criscitiello et al., "Bowel obstruction and peritoneal carcinomatosis in the elderly. A systematic review," Aging Clinical and Experimental Research, vol. 29, no. 1, pp. 73-78, 2017.

[149] M. Santangelo, G. Vescio, L. Sommella et al., "Extended total gastrectomy: What indication in 3rd millennium," Minerva Chirurgica, vol. 56, no. 1, pp. 1-6, 2001. 


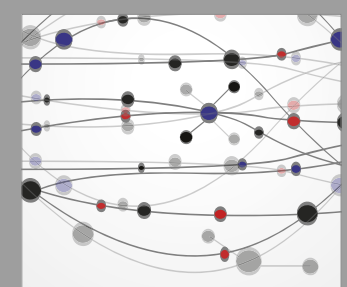

The Scientific World Journal
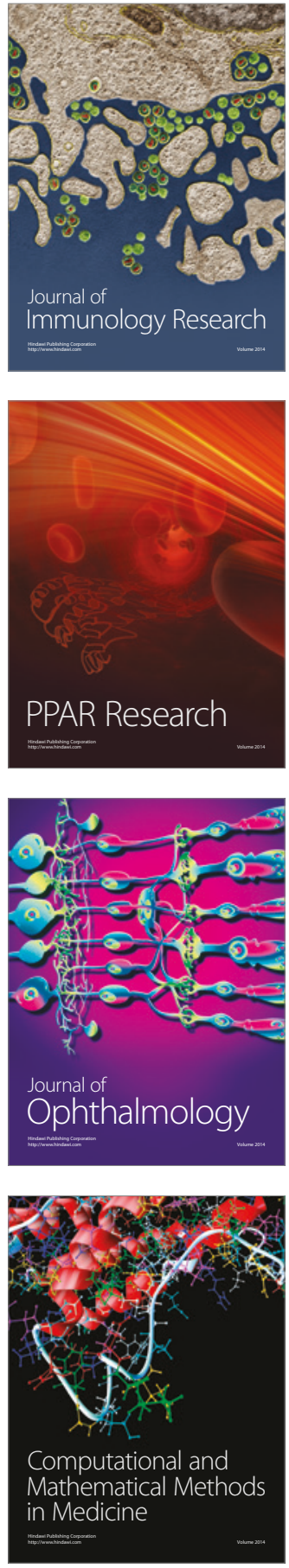

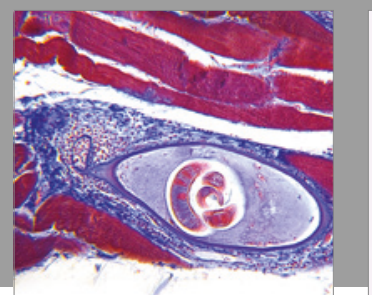

Gastroenterology Research and Practice
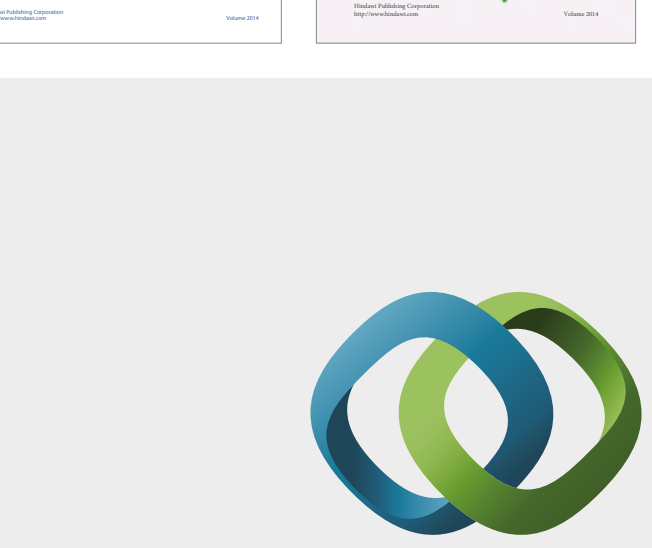

\section{Hindawi}

Submit your manuscripts at

https://www.hindawi.com
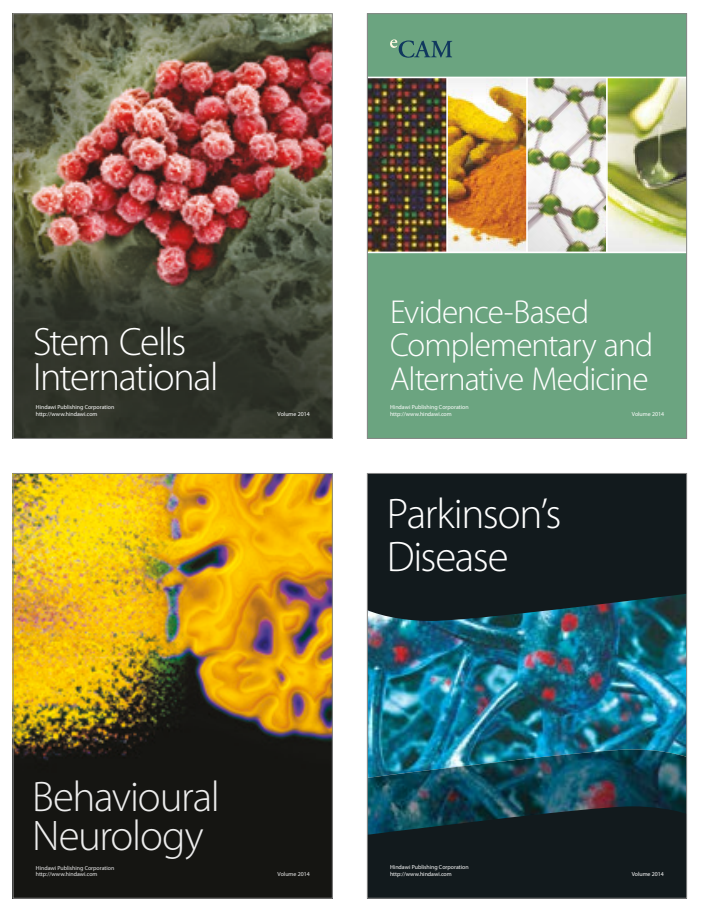
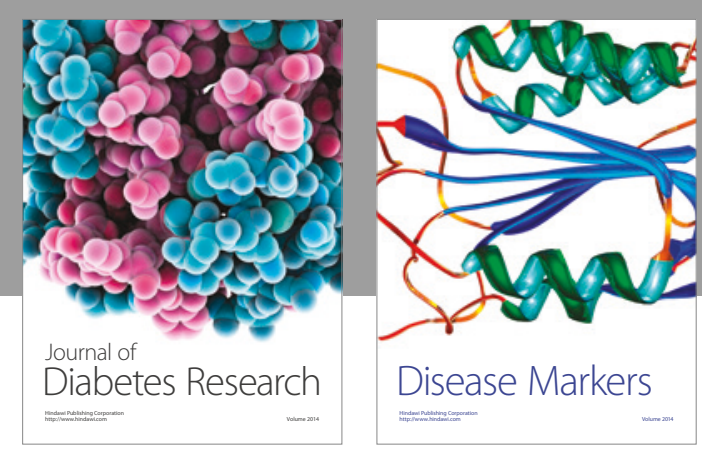

Disease Markers
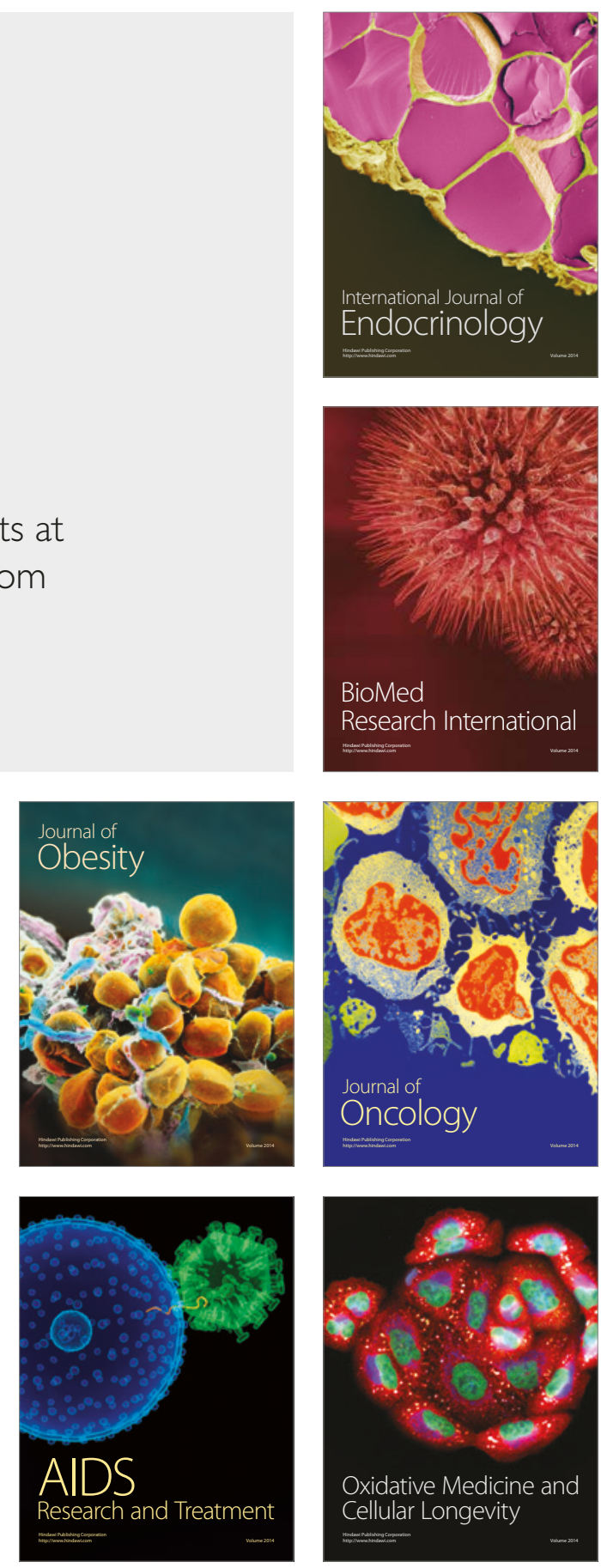